\title{
A Framework for Multiple and Heterogeneous Earthquake Disaster Information Fusion*
}

\author{
Jinghai Xu \\ College of Geomatics Engineering \\ Nan Jing University of Technology, Nan Jing, China \\ Email: Xu_jing_hai@163.com \\ Gaozong Nie \\ Institute of Geology \\ China Earthquake Administration, Beijing, China \\ Email: niegz@ies.ac.cn
}

\begin{abstract}
To realize the fusion of multiple and heterogeneous earthquake disaster information and get effective disaster information, situation and characteristics are addressed on different earthquake disaster information acquiring methods, in which some new earthquake disaster information acquiring methods are described. A fusion architecture of multiple and heterogeneous disaster information is put forward, including data level, fusion level and application level. And function principle of each level is described. Finally key technologies and realization methods in the fusion architecture are expounded, which are transformation and match of multiple disaster information and coding of the information.
\end{abstract}

Index Terms - earthquake disaster information, earthquake emergency, multiple source data, information fusion, GIS

\section{INTRODUCTION}

Earthquake with unexpected and transient features is one of the inevitable natural disasters for human. Earthquake disaster is a serious threat to human security, causing heavy casualties and economic losses, such as the Wenchuan earthquake in 2008, Yushu earthquake in Qinghai in 2010. The earthquake emergency and rescue is one of the important ways to effectively reduce earthquake disaster, and have good results in real disaster [1-2]. But it is one of the key issues to restrict the earthquake emergency rescue that the lack and delays of disasters information after the earthquake. From so many earthquake emergency rescues, it can be found that the delay of disaster information acquiring could often have bad effect on rescue. And effective relief operations cannot be made against losses for different areas and the actual needs of the earthquake zone, at the same time disaster relief commander is difficult to make quick decisions. In recent years, with the advancement of earthquake emergency researches and related technologies, getting earthquake disaster information rapidly is becoming a hot topic of earthquake emergency research. In the field of earthquake disaster information collection and rapid assessment, many countries have successful exploration, in which Japan and the United States get much achievement [3]. Because of the delaying of getting disaster information, the government has suffered public criticism and blame in Japan's Kobe earthquake. Then after the earthquake, the National Land Agency is responsible for developing a special earthquake disaster prevention information system (DIS), designing to access disaster information quickly[4]. In "Earthquake Emergency Response Planning" of United States, much emphasis has been attached to disaster information collection and transmission, which ensuring to obtain accurate disaster information and smooth information transmission channel in the shortest time after earthquake [5]. With the efforts of all levels of government and researchers, a variety means of disaster have been initially established in China, such as remote sensing, phone report, SMS disaster collection and so on. However, the received disaster information has large volume, different types and complex contents. Then it is important in earthquake disaster acquiring that how to implement these multiple and heterogeneous disaster to obtain effective disaster. It will be discussed in this paper.

\section{ANALYSIS OF MULTIPLE ACQUISITION METHOD OF EARTHQUAKE DISASTER INFORMATION}

\section{A. Remote sensing based earthquake disaster information acquiring method}

In various disaster information acquiring technologies, remote sensing based method is one of mostly studied and widely used methods. This method can be dated back to 1906. Lawrence used kites as platform, carrying a camera, successfully captured the scene of the San Francisco earthquake[6]. With the rapid development of remote sensing technology, there are many researches and application in domestic and foreign, which has already been applied in several real earthquakes, for example, Niigata earthquake in Japan, Chi-Chi earthquake in Taiwan. After the 2008 Wenchuan earthquake, various remote sensing platforms have been applied and gotten a good result in earthquake disaster accessing. After the earthquake, the National Disaster 
Reduction Committee Office has received a lot of remote sensing data, accumulated up to 1277 maps from 24 satellites of 12 countries [7].

By using different standards, remote sensing based earthquake disaster information acquisition technologies can be divided into the following categories. According to different phase of image, it can be divided into singlephase damage recognition technology and identification technology temporal damage. According to different algorithms, it can be divided into point feature criteria algorithm and regional analysis algorithm; According to the spectral characteristics of remote sensing images, it can be divided into visible light remote sensing image and non-visible light remote sensing image. Each technology has its own characteristics, and constrained by its own technology. For example, multi-temporal remote sensing damage recognition technology should have high recognition accuracy in theory, but its must have two images with high registration accuracy and correlation matching accuracy. Single-phase technology required images with less phase, but generally more suitable for high-resolution remote sensing images (including aerial photos).

In recent years, the development of photogrammetric and remote sensing technology provides better opportunities and prospects for remote sensing based disaster acquisition method, such as SAR image based method, small aircraft based method and laser scanning based method. However, the current remote sensing based remains to be further improved in terms of algorithm speed, accuracy and so on.

\section{B. SMS and Internet based earthquake disaster information acquiring method}

The template is used to format your paper and style the text. All margins, column widths, line spaces, and text fonts are prescribed; please do not alter them. You may note peculiarities. For example, the head margin in this template measures proportionately more than is customary. This measurement and others are deliberate, using specifications that anticipate your paper as one part of the entire proceedings, and not as an independent document. Please do not revise any of the current designations.

SMS and Internet based earthquake disaster information acquiring methods are appeared and developed rapidly in recent years. The famous system of "Did you feel it", developed by the U.S. Geological Survey, collects intensity evaluation data provided by the public with the Web technology and make into digital maps, so the scope of the earthquake can be quickly calculated [8-9]. Dumfries Earthquake Investigation Bureau has also used Internet technology to obtain seismic data provided by the public, similar study is being researched by related departments in Germany[10]. In Japan, Y. Hisada and M. Murakami also studied earthquake information getting method by using Internet, GSM and other technical through the public and government, which achieved good results in an earthquake emergency drill[11]. There are also similar research being carried out in china, such as Internet based seismic hazard information acquisition technology studied in literature [12], and research on earthquake information publishing software based on SMS[13-15].

"The work outline of earthquake monitoring and prevention", issued by China Earthquake Administration, requests city and county building of earthquake quick reporter network, which lay a foundation for SMS and Internet based disaster rapid report method, and make this method be an important method of disaster getting in the future. China Earthquake Administration released 12322 public service platforms for getting earthquake disaster information in 2009. After the earthquake, mobile phone users anywhere in the country can send messages about their known to 12322 in required format, so the feedback disaster information can be rapidly collected by the 12322 platform.

As a new disaster information collection method, the method should be improved and researched in many aspects. Such as, without a mature work flow to quickly report disaster information; how to scientifically and practically choose the contents of the disaster information for report; how to strengthen integration and interaction between disaster information collected and existing information. However there are some explorations in this filed at present[13-14].

\section{Voice based earthquake disaster information acquiring method}

Telephone is one of the mostly rapid ways for victims in the earthquake damage area to directly report disaster information to the earthquake administrate department. It is a new method to collect earthquake disaster information thought extracting and analyzing received voice information. In this method related information is extracted, and then information on the disaster scene is obtained.

Voice based disaster collection method can be in two modes: one is voice processing mode, disaster reporting staffs report disaster information by phone in voice, then the voice signals through Modem access the computer system. In computer voice signals are received by voice processing system, then turning into text data, saving into the voice text database, and analyzing by data analysis system, so useful disaster information is gotten. Another is disaster information response mode, in this model, according the directing of the response system, disaster reporter use phone keyboard to select disaster type and severity for disaster information reporting. The disaster reporters' phone keyboard input are received through the voice card by disaster response system, then the degree of disaster is determined based on their input. Data received are saved into the disaster database for analysis system.

Automatic processing of computer can be achieved thought voice processing mode, which simplify the operation of disaster reporters, improve reporting efficiency. But there are some drawbacks, for example, a caller must report in a standard mandarin. As the speech recognition error rate is high, the callers with nonstandard pronunciation may affect the information extraction and analysis. Identifying information is usually untreated documents, which need to be analyzed. Phone 
disaster response system can solve the above problems in some extent through the voice direction and interaction with the disaster reporting staffs. However, it needs the disaster reporters' complex operation in the emergency, which may impede the collection of disaster information.

12322 public earthquake disaster reduction service platform provides not only the SMS services but also a 12322 hotline service. Currently, the hotline service combines above two modes. Some cities have realized local artificial services, such as Beijing, Hebei. A considerable amount of research work is still needed on how to improve speech recognition in the disaster information, strengthen disaster automated collection, reduce interactive of voice direction in the future.

\section{SYSTEM ARCHITECTURE OF MULTIPLE AND HETEROGENEOUS DISASTER INFORMATION FUSION}

To fuse multiple and heterogeneous disaster information, disaster information fusion system architecture is designed, and is shown in figure 1. In this architecture, there are three levels: data level, fusion level and application level.

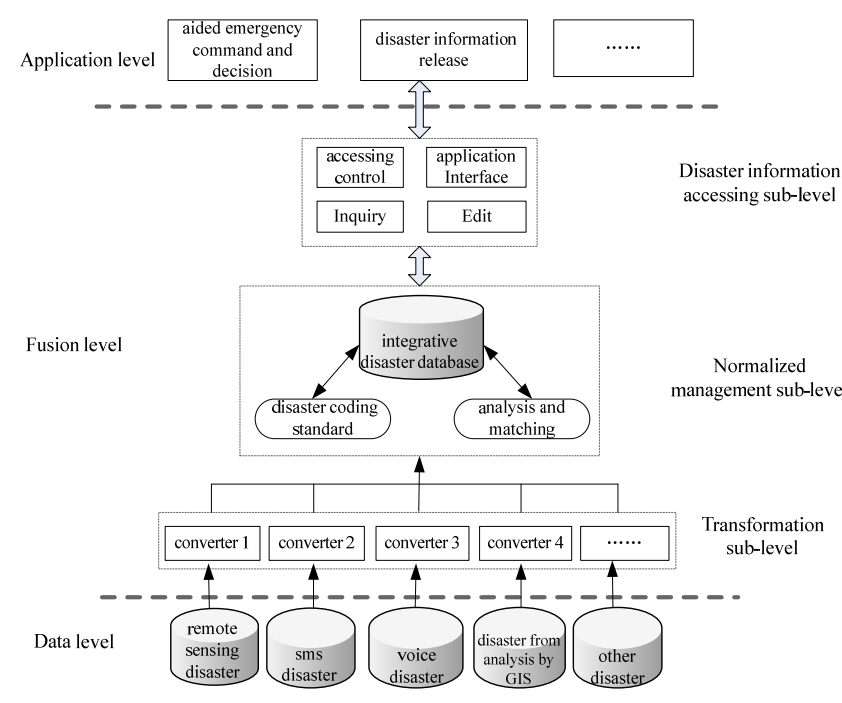

Figure 1. Structure of multiple disaster information fusion

\section{A. Data level}

The aim of this level is to obtain original disaster information by various ways, and it is the lowest level of the architecture. The ways to get the disaster information include remote sensing, SMS, voice way, the simulated disaster information produced by GIS, as well as disaster information getting from other ways, such as reported files, reported fax. In this level, there are many differences of this information in data formats, data representations and data contents. For example, remote sensing disaster information may be expressed by raster format, and GIS based simulated disaster information are expressed by vector format, and reported disaster documents are more expressed in the unstructured text form. In order to realize the fusion of them, they firstly need to be converted and processed.

\section{B. Fusion level}

Fusion level is devoted to realize the conversion, matching and integration of multiple and heterogeneous disaster information and manage integrated disaster information to provide effective support for application level by controlling the accessing of disaster information. The level is composed of three sub-levels, which includes conversion level, normalized management level and disaster accessing control level.

The goal of conversion level is to achieve the conversion and processing of the disaster information from different sources, and provide the basis for data fusion. Different data transformation methods should be adopted, according to different disaster information. For example, data format of remote sensing base disaster information need to be transformed and SMS based disaster information need be converted from text format information to GIS-based disaster information. Conversion level is formed by a number of converters, and through different converter, different disasters can be analyzed and transformed. So it could make basis for normalized management of the disaster information. The detail realization method of converters is shown in figure 2, which will be further introduced in the following section.

The aim of normalized management level is to achieve the unified expression and management of the multisource disaster information. As shown in figure 1, the sub-level is composed of disaster information standards coding module, analysis matching modules of different disaster information and integrated disaster database. Disaster information standards coding module is the key for fusion. Disaster encoding method need not only consider disaster information itself, but also should take into consideration the integration with other information's encoding method. Analysis and matching modules is mainly used to realize disaster information match from different sources. For example, for the disaster on the same site, some may be described by $\mathrm{X}, \mathrm{Y}$ coordinates and some may be described by some close building or the road intersections. To fuse disaster information from different sources, the information should be analyzed and matched. Finally, after matched of the multi-source disaster information, it will be managed and stored by spatial database, so integrated disaster database is formed.

After normalized expressing and managing multisource disaster information, effective application of the information can be achieved in earthquake emergency on the support of disaster accessing control sub-level. This sub-level includes disaster information query and edit modules, accessing control module and application interface module. Thereinto, query and edit modules achieve disaster information query, search and other functions. Accessing control module and application interface module provide the basic interface functions for 
the disaster information applications, such as acquiring disaster information, analyzing disaster information.

\section{Application level}

Based on the fused disaster information, this level achieves the application of the disaster information and servers for earthquake emergency and rescue. The disaster information can be used for auxiliary earthquake emergency and command. Through the analysis and application of disaster information can greatly improve the pertinence and efficiency of earthquake emergency and rescue, for example rapidly analyzing earthquakeaffected areas, getting the distribution information about aid needed disaster victims, and through analyzing the range of houses damage determining the focus scope of emergency search and rescue. Meanwhile, the level could provide effective disaster information to release. Releasing disaster information to the media and the public is helpful to stabilize the emotion of social public and get assistance from the worldwide.

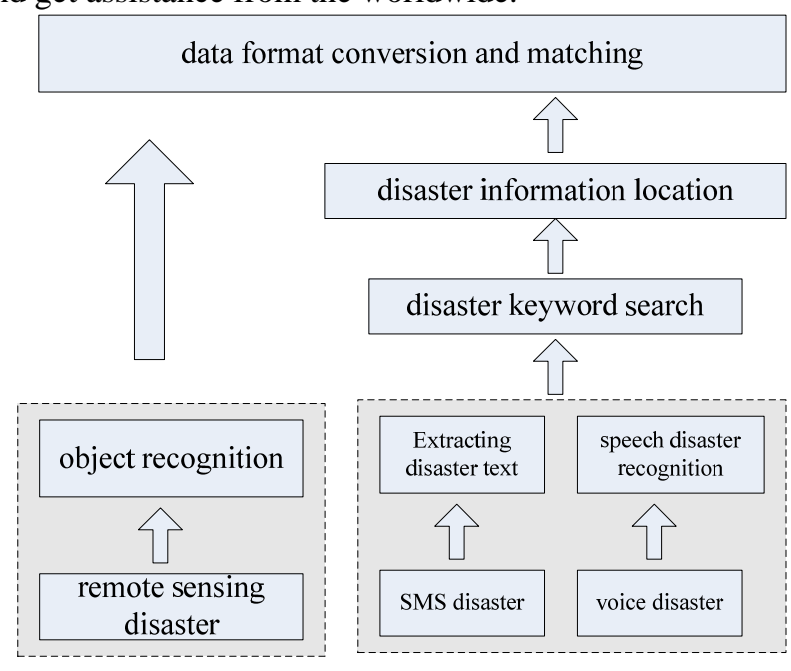

Figure 2. Transform of multiple disaster information

\section{KEY TECHNOLOGIES OF MULTIPLE EARTHQUAKE DISASTER INFORMATION FUSION}

\section{A. Transformation and matching of multiple earthquake disaster information}

Multi-source earthquake disaster information from has a number of data formats and quantity, such as manual data (SMS, phone voice information, fax information), document data, video data and structured data (the data existed in system database). In order to realize the fusion of multiple and heterogeneous disaster information, transformation and matching of multiple disaster information is one of the key technologies. The transformation includes two levels conversion: data structure level conversion and semantic level match.

The multiple and heterogeneous disaster information need to be transformed on data formats. There are a lot of studies on transform of structured data, such as transform between raster data format and vector data format. So this conversion can be realized by a large number of tools, such as FME, Arctoolbox. The conversion of remote sensing belongs to this conversion, whose process is: firstly, recognizing earthquake information from remote sensing images. Then, through data format conversion, transform the information from raster data format to uniform disaster data format (such as vector data format and other requirements raster format).

Besides the conversion between structured data, it is a difficult thing that how to convert SMS data and voice disaster data to spatial format data. As shown in figure 2, in the process of transforming SMS format disaster information, firstly it extract effective disaster information from a large number of received disaster short messages, and get effective disaster SMS text. Then searching for disaster information according to the disaster keywords such as seismaesthesia, casualties, and the retrieved disaster information are expressed and coded in standardization. Finally, according to supplementary information related to the disaster SMS determine the location of earthquake, such as sending number and time, and the integration of disaster messages and geographic information is realized. So the SMS messages are converted to structured disaster geographic information. The disaster location can also be determined by adding appropriate code in earthquake disaster short message, so the disaster location can be reported by disaster reporting staff, when they report disaster information by short message. The keywords searching of disaster information plays an important role in the conversion process. Semantic web technologies can be used to achieve search and extraction of earthquake disaster information by selecting appropriate keywords.

The method of the conversion of speech disaster information similar to message disaster, also firstly need to convert them into disaster text messages, and then using the similar method of short message disaster to achieve its transformation. In this process speech recognition technology is the key, the research of this technology began as early as 1920, and with many years of research, it has been applied in many fields. Overall, there are three ways of the speech recognition currently: method based on channel model and speech recognition, method based on template matching and method by using artificial neural network technology. But many aspects of speech recognition technology have certain limitations which need to be further developed, such as the algorithm models, which is difficult to make computers really understand human languages. And in the robustness, the multi-languages mixed recognition and unlimited words recognition and other aspects need further improvement. But it can be expected, with the development of speech recognition technology, some related methods of the collection of disaster messages which are based on speech recognition will further improve and enhance its usability.

The conversion of multi-source disaster information and matching are closely related, and data conversion is the basis of the matching. To achieve the fusion of multi- 
sources disaster information, it also needs to achieve the matching of different disaster information. For example, if a place where ground subsidence occurred after the earthquake, there may be different disaster information acquiring methods. Such as the speech disaster information may described the disaster information based on a major sites and the GIS data may be based on geographic coordinates. When matching multi-source information, the multi-scale map matching technology which is commonly used in spatial information field can be used for reference. That method is: firstly, unifying and converting the spatial framework of multi-sources disaster information, that converting different spatial coordinates into a unified coordinate system, so that all the disaster information expressed by a uniform basis. Secondly, respectively, in a multi-source disaster, selecting the appropriate number of control points, and matching the multi-source disaster information through the control points in a unified geographical coordinates. Then matching the control points, and through matching them with each other to calculate the conversion parameters between the different disaster information. Finally, using the calculated transformation parameters, it can achieve the matching between different disaster information.

\section{B. The classification of multiple earthquake disaster information}

Earthquake disaster information includes earthquake casualties, property losses, the destruction of environmental and social function, etc. After an earthquake, the disaster information from disaster zone has the characteristics of various kinds, multiple sources and many diverse forms. It needs follow basic principles of information classification to establish a reasonable disaster information classification system. The principles are: the classification should consider the inheritance and continuity with domestic seismic information or other relative information; scientific principle: the classification system should be an orderly disaster information classification system, according to the objective essence attribute of the disaster information; practical principle: the classification of disaster information should be able to directly server for earthquake emergency command and rescue, disaster investigation and assessment, information coding, information collection and distribution; scalable principle: for the rapid development of the disaster information investigation technology and earthquake emergency rescue technology, it should be have expansion ability for new technology application and new needs.

Information classification methods are including: line classification method, surface classification method and combination or hybrid classification method [16-17]. Line classification method is also called level classification method, in this method information will be divided into several levels according to the selected several attributes (or characteristics). Same level of a branch has the coordinate relationship and the relationship of different levels is subjection. According to inherent several attributes (or characteristics) of the given taxonomy object, surface classification divides it into a set independent categories. Each group of the categories constitutes a "surface", and each "surface" of no subordinate relationship will be parallel aligned with a certain order. This method is adopts of "name code of cadres”, national standards GB / T 12403. Mixed classification method combines the line and surface classification methods, one as priority and another as supplement. According to the characteristics of multiple sources information, the third classification method is chosen in this paper, line classification as priority and surface classification as supplement in the mixed classification.

Considering the characteristics of multiple sources, the earthquake disaster information has been divided into seven classifications. They are

(1) seismaesthesia information: feel of people, such as slight feeling, strong feeling.

(2) Casualties information: injured and death person, such as people death number, people injury number.

(3) House damage information: house type , damage extent.

(4) Secondary disaster information: Secondary geological disasters, poison, explosion, fire, flood, etc, and the information includes disaster scale and location.

(5) Lifeline information: water supply, gas supply, drainage, power supply, traffic, communication, etc, and the information includes severity, affect scale.

(6) Social influence information: the comprehensive influence of earthquake (affect scale of social organizations, social life order, work order, production order).

(7) Other information: other related earthquake disaster information.

\section{The coding of multiple disaster information}

The coding of disaster information is critical in matching and fusing multi-source and heterogeneous information. In theory, under the guidance of a certain standard, the code should be only for the same disaster information. But in fact, as the disaster information getting with different methods, as well as understanding the same disaster information with different angles in the disaster collection system, it may appear the situation that different information have same code or the same information has different codes. If directly using the multi-source disaster information in an emergency command system, the system must easily lead to confusion. Therefore, when discussing the integration of disaster information, it needs study the code of disaster information.

Objectively, for a variety of information and data, it must be classified and coded according to some rules so that it can be uniform and orderly collected into the computer, and to achieve its integration and management. Storing and searching them by category to meet the needs 
of various applications. Otherwise, the multi-source heterogeneous data which are collected by different people or different departments could not be standardized and aggregated due to the nuances in definitions, concepts, units of classification aspects. And after these information are stored into the database by the business sector in accordance with their methods, they may be a pile of messy data. It is difficult to find or retrieve the data which are inconsistent with the requirements and it may even lose value for database [18].

At present, there are main three information coding methods in china: tree structure coding, chain coding and hybrid structure coding. Tree structure is characterized by a subordinate relationship between the code points, containing more information with fewer code bits, but it can not be easy coded and identified. In chain structure, the number of bits of each code has independent meaning, which contains less information than the tree structure, but the structure is simple and easy coded and identified. Hybrid structure is a combination of these two coding system, with the common advantage of single code structure and multi-code structure.

The coding of multi-source earthquake information can refer to geographical information coding method, and the existing information coding method in earthquake emergency field, through the expansion of tree coding, forming the disaster information coding. Taking into account the information coding scalability, compatibility and other principles, the disaster information coding is shown in Figure 3. This code is organized by classification, composed by a total of six numbers from the three grades, and each grade has two number codes. Each grade is the classification of earthquake disaster information, the first grade means disaster information acquiring methods (such as short message based method), the second grade means the disaster information categories (such as the lifeline system disaster information), the third means detail disaster information (such as XX bridges damaged). This coding is practical and simple, and it can be easily identified, and it can also reduce the work of data processing and code conversion.

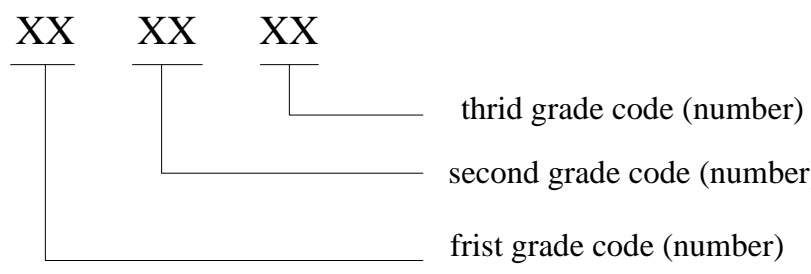

Figure 3. Illustration of disaster information coding

\section{CONCLUSIONS}

Since the State Council earthquake relief headquarters are being constructed in 2000, earthquake emergency systems cluster have been built, in which the State Council earthquake relief headquarters is the lead, 31 provincial headquarters are the centers of districts, and on-spot command system is the front. The system cluster provides sites and necessary technical for government's earthquake emergency command and relief. However, information collection and processing capability after the earthquake still need to be improved in China. In this paper, the fusion of earthquake disaster information with variety types and large quantities is researched. Methods of obtaining earthquake disaster information based on remote sensing, on SMS and Internet and on voice are analyzed. Current situation and characteristics of the development of various collection methods are discussed, and the existing problems of these methods are pointed out. A three level fusion architecture of multiple and heterogeneous disaster information is proposed, which contains data level, fusion level and application level. Then the working principle of each level is described in detail. Finally, key technologies and realization methods $f$ multiple and heterogeneous disaster information fusion are discussed: conversion and matching as well as coding of multiple and heterogeneous disaster.

\section{ACKNOWLEDGMENT}

This work was supported in part by a grant from the National Natural Science Foundation of China (Grant No. 40901272), National Key Technology R\&D Program of China (Grant No. 2006BAC13B03, 2008BAK50B03).

\section{REFERENCES}

[1] Tang Aiping, Xie Lili, Tao Xiaxin. Application of GIS to build earthquake emergency response system for urban area[J].Journal of Harbin Institute of Technology(New Series),2002,9(1):38-42.

[2] Ma Haoran, Feng Qimin, MO Shanjun. Research on the decision support system of the urban seismic emergency. world earthquake engineering,2005,21(1):30-36.

[3] Zongjin Ma. China's Earthquake Disaster Mitigation Engineering [J]. Disaster,2005,20 (2):1-5.

[4] Tang Xiao-five, Kato Takaaki, small out rule. Japanese disaster response system [M]. Beijing: Chinese Building Industry Press,2003.

[5] Federal Emergency Management Agency (FEMA). Guide for All Hazard Emergency Operations Planning[R], 1996, FEMA, Wash-ington DC, USA.

[6] Jiahang Liu, Yang Jianfeng, Chengjie Wei, et al. Remote sensing for earthquake damage information technology, history, current status and trends of [J]. Natural Disasters, 2004,13(6):46-52.

[7] Yida Fan, Siquan Yang, Chengjie Wei, et. Wenchuan Earthquake monitoring and evaluation method [J]. Remote Sensing,2008,12(6):858-863.

[8] USGS. Did You Feel It? Community Internet Intensity Maps[EB/OL]. URL: http://earthquake.usgs.gov/ eqcenter/dyfi.php.

[9] USGS. Did You Feel It? Citizens Contribute to Earthquake Science[EB/OL]. http://pubs.usgs.gov/fs/ 2005 /3016/cf.php.

[10] Haubrock S, W ittkopf T, Grünthal G,et al.Communitymade Earthquake Intensity Maps using Google s API [C].10th AGILE International Conference on Geographic Information Science, AalborgUniversity, Denmark, 2007: 1-5. 
[11] Hisada Y, Murakami M, Zama S. Quick collection of earthquake damage information and effective emergency response by collaboration between local government and residents. The 14th World Conference on Earthquake Engineering October 12-17, 2008, Beijing, China.

[12] Xiang Dong, Lanxi Xiao, Xiansong Du, et al. North Chinese Earthquake Sciences [J]. 2007,25(3):6-10.

[13] Shengguo Liu, Jingchun, Youwen Wu. Messaging platform based on the earthquake quick report released Implementation [J]. Seismological and Geomagnetic Observation and Research, 2006,27(S1):116-119.

[14] Jinghai $\mathrm{Xu}, \mathrm{Xu} \mathrm{Xu}$, Weiqing Liu. Nanjing earthquake quick report system Based on GIS/GSM [J]. Nanjing University of Technology (Natural Science),2009,31(1):101-105.

Jinghai Xu, was born in JingZhou city, China, in 1977.4. He received B.S degree in survey engineering and M.S degree in civil engineering in NanJing University of Technology, China in 2000 and 2003. Then he received $\mathrm{Ph}$. D degree in photogrammetry and remote sensing in Wuhan University, China in 2003.

Now he is an associate professor of College of Geomatics Engineering NanJing University of Technology. His published articles include:

Jinghai $\mathrm{Xu}, \mathrm{Xu} \mathrm{Xu}$, Gaozong Nie, et al. Earthquake emergency situation plotting technology based on GIS. Geomatics and Information Science of Wuhan University, 2011,36 (1):66-70.

Jinghai Xu, Yang Yan, Minxian Deng, et al. GIS-based quick report and eatimation of earthquake disaster information. Journal of natural disasters, 2010,19(4):141-146.

Jinghai Xu, Qingquan Li. Research on navigable digital map data increment update based on temporal GIS. Geomatics and Information Science of Wuhan University, 2008, 33 (11):11821185.

His research interests are focused on spatial information based urban disaster mitigation and hazard prevention.

Gaozong Nie was born in 1964.1. He is a professor of Institute of Geology China Earthquake Administration. His research interests are earthquake emergency, natural hazard prevention.
[15] Xianghua Shuai, Jiansheng Hou, Qin Liu. Site discrete points based on seismic hazards in the disaster reports of simulation [J]. Earthquake Research,2009,31(2):321-333.

[16] Du Wei. Research about rural socio-economic statistical information classification and coding standards. Journal of Anhui Agriculture Science, 2010, 38(23):12846-12847.

[17] Zheng Kui,Shu Xueming, Yuan Hongyong, et al. On Classfication method of network public opinion triggered by incidents. Computer Applications and Software, 2010, 27(5):3-6.

[18] Bai Xianfu, Li Yongqiang, Chen Jianhua, et al. Research on earthquake spot emergency response information classification. Journal of Seismological research, 2010, 33(1):111-118. 